\title{
El uso de las redes sociales como estrategia educativa.
}

The use of social networks as an educational strategy.

Efraín Velasteguí López. ${ }^{1}$

\section{Resumen.}

La influencia de las redes sociales en la sociedad gracias al Internet ha cambiado tanto nuestras vidas que no podemos mirar hacia otro lado. Y las redes sociales, que están en boca de todos, han venido para quedarse. Por ello, es importante que en los centros educativos se conozcan y se usen de forma que los alumnos puedan beneficiarse de ellas para sus estudios y desarrollo personal donde también puede ser un medio de comunicación para realizar al docente como apoyo de investigación, por otro lado llegar a conocer personas con un amplio conocimiento que le ayudara.

Uno de la principal red social a nivel mundial Facebook han sido analizados para su aplicación tanto en adolescentes como en el ámbito universitario, destacando su potencial como complemento a la docencia. Estas tecnologías permiten a los alumnos interactuar con los contenidos y comunicarse a través de diferentes medios, seleccionarlos, remezclarlos, crearlos y compartirlos.

Para ampliar más el tema de la influencia que a tenido el uso de las redes sociales como medio educativo es el estudio actual donde sea beneficiado no solo a los estudiantes y docentes sino también a los padres para que puedan llevar el control y estén informados del progreso académico de su hijo.

Las redes sociales único medio de influencia a nivel mundial que ha cambiado el modo de vidas y facilitando la comunicación de las personas, podemos hablar mucho sobre los beneficios, pero también tienes sus desventajas.

Palabras claves: Red Social, Educación, Investigación, Desarrollo Personal, Tecnologías.

\section{Abstract.}

The influence of social networks on society thanks to the Internet has changed our lives so much that we can not look the other way. And social networks, which are on everyone's

\footnotetext{
${ }^{1}$ Ciencia digital, Ambato, Ecuador, luisefrainvelastegui@ cienciadigital.org
} 
lips, have come to stay. For this reason, it is important that in educational centers they are known and used in such a way that students can benefit from them for their studies and personal development where they can also be a means of communication to make the teacher as research support, on the other hand Get to know people with extensive knowledge that will help you.

One of the main social networks worldwide, Facebook, has been analyzed for its application both in adolescents and in the university environment, highlighting its potential as a complement to teaching. These technologies allow students to interact with content and communicate through different media, select them, remix them, create them and share them.

To further expand the issue of the influence that the use of social networks has had as an educational medium is the current study where it benefits not only students and teachers but also parents so that they can take control and be informed of progress academic of your children.

Social networks are the only means of influence worldwide that have changed the way of life and facilitating the communication of people, we can talk a lot about the benefits but also have their disadvantages.

Keywords: Social Network, Education, Research, Personal Development, Technologies.

\section{Introducción}

\section{La red social}

La Primera Red Social creada sobre estas nuevas tecnologías de la información y de las comunicaciones fue"classmates.com"que fue creada por Randy Conrads, con esta red social se pretende que la gente pueda recuperar o mantener el contacto con antiguos compañeros del colegio, instituto, universidad, etcétera

Grafica 1: Primera red social para la interacción de profesores con estudiantes

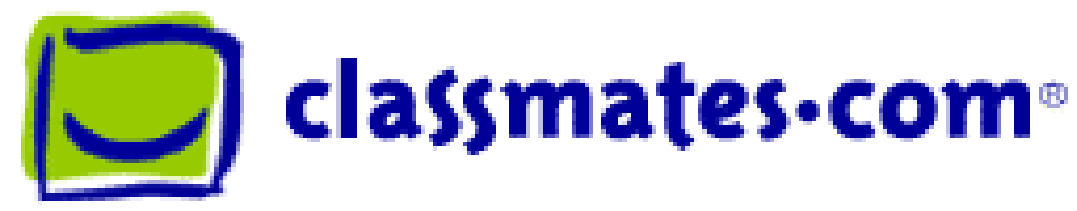

\footnotetext{
Autor: Emiliano Leyva.

Fuente:http://2.bp.blogspot.com/hJH2T3RBQL8/ThHIBTJtuxI/AAAAAAAAB4I/AqjVPp9i8a0/s1600/cm_logo. gif
} 


\section{Las redes sociales más conocidas}

Facebook es un sitio web de redes sociales creado por Mark Zuckerberg y fundado junto a Eduardo Saverin, Chris Hughes y Dustin Moskovitz.

Gráfica 2: Conoce las redes sociales más utilizadas.

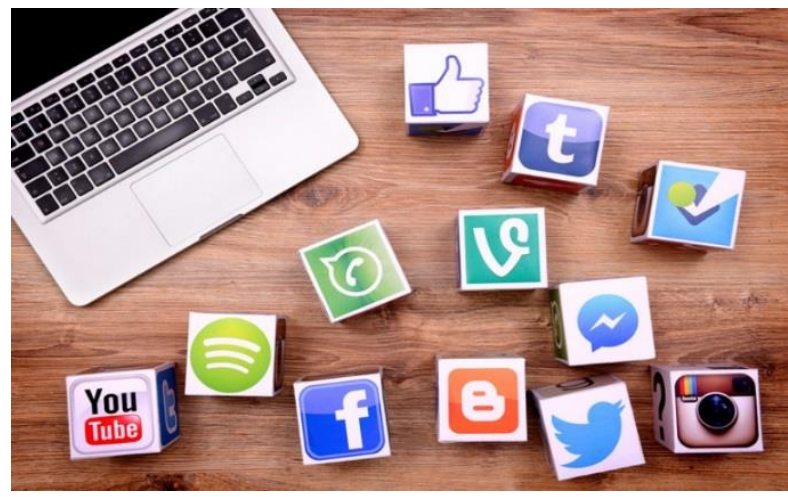

Autor: Sheyla Carralón

Fuente: https://expansion.mx/empresas/2018/01/09/las-redes-sociales-dominanel-uso-de-internet-en-telmex-televisa-y-megacable

YouTube es un sitio web en el cual los usuarios pueden subir y compartir vídeos. Fue creado por tres antiguos empleados de PayPal en febrero de 2005 Google Inc. es una empresa multinacional estadounidense especializada en productos y servicios relacionados con Internet, software, dispositivos electrónicos y otras tecnologías.

Instagram es una red social y aplicación para compartir fotos y vídeos. Permite a los usuarios aplicar efectos fotográficos como filtros, marcos, colores retro y vintage, y posteriormente compartir

WhatsApp es una aplicación de mensajería instantánea de pago para teléfonos inteligentes, para enviar y recibir mensajes mediante Internet, complementando servicios de correo electrónico, mensajería.

Tumblr es una plataforma de microblogging que permite a sus usuarios publicar textos, imágenes, vídeos, enlaces, citas y audio a manera de tumblelog. Su sede está en Manhattan (Nueva York, Estados Unidos) y fue fundada por David Karp en el año 2007. (Alejandra, 2015)

\section{Concepto de Red Social}

Según (Fernández R. 2013., Flores J. 2014., Harold H. 2014., Requena F. 2013., Rouse M. 2017. \& Torre L. 2012) afirman que una red social se define como un servicio que permite a los individuos construir un perfil público o semipúblico dentro de un sistema delimitado, articular una lista de otros usuarios con los que comparten una conexión, y ver y recorrer su lista de las conexiones y de las realizadas por otros dentro del sistema. La naturaleza y la nomenclatura de estas conexiones pueden variar de un sitio a otro. Estas autoras han desarrollado un artículo en el cual se detalla la historia de las redes 
sociales, desde la aparición del primer sitio reconocido de red social en 1997, denominado SixDegrees.com hasta la apertura de Facebook (everyone) en el 2006. En este artículo, también se incluyen referencias de investigaciones sobre el tema de la privacidad en las redes sociales relacionadas con la seguridad, las amenazas potenciales para los más jóvenes, entre otras.

Las redes sociales son lugares en Internet donde las personas publican y comparten todo tipo de información, personal y profesional, con terceras personas, conocidos y absolutos desconocidos", afirma Celaya (2008). Por su parte, Wikipedia la define como: "una estructura social que se puede representar en forma de uno o varios grafos donde nodos representan individuos y las aristas las relaciones entre ellos.

\section{Tipos de redes sociales}

Según Celaya (2008), existen tres clasificaciones principales de redes sociales:

1. Redes profesionales (por ejemplo, LinkedIn, Xing, Viadeo)

2. Redes generalistas (por ejemplo, MySpace, Facebook, Tuenti, Hi5)

3. Redes especializadas (por ejemplo, Ediciona, eBuga, CinemaVIP, 11870)

\section{Las redes profesionales}

Este tipo de redes han contribuido a fomentar el concepto de "networking" entre las Pymes y mandos intermedios en el caso de las empresas más grandes. Su valor agregado es que permiten ir desarrollando una amplia lista de contactos profesionales, tanto para intercambios comerciales como para interacción y búsquedas de oportunidades entre las personas. Normalmente, los usuarios que ingresan a estas redes incluyen una descripción de su hoja de vida, lo cual constituye el punto de partida en sus interacciones posteriores. Todas estas plataformas cuentan con un buscador interno que nos permite rastrear la base de contactos por el nombre de la persona que buscamos o por el nombre de la empresa o universidad. Los usuarios a su vez pueden integrarse entre sí de acuerdo con sus expectativas, gustos y preferencias.

¿Conoces Facebook, Instagram, Youtube o Twitter? Seguro que sí. ¿Te suenan también Snapchat, LinkedIn, Tumblr o WeChat? Puede que también. En todo caso, ;acertaste! En esta página y las siguientes hablamos de Redes Sociales de Internet.

Los humanos siempre nos hemos relacionado por grupos: familiares, laborales, sentimentales, etc. En una red social los individuos están interconectados, interactúan y pueden tener más de un tipo de relación entre ellos.

\section{Redes Sociales}

En la actualidad, el análisis de las redes sociales se ha convertido en un método de estudio en ciencias como la antropología o la sociología. Internet y las nuevas tecnologías favorecen su desarrollo y ampliación.

En este sentido, debemos tener en cuenta que todo lo que se publica en una red social queda registrado. Es importante controlar qué contenidos vamos a mostrar y qué 
impresión queremos causar. Hay que poner especial atención y cuidado, no solo en la configuración de nuestra privacidad, sino también en el estilo, la claridad y la educación con que nos expresamos.

\section{Redes sociales generalistas}

Este tipo de espacios cuentan con perfiles de usuarios muy similares a los anteriores, pero con ritmos de crecimiento distintos, marcados por generación de contactos, quienes ingresan con el fin de ponerse en contacto con personas cercanas y no tan cercanas, para comunicarse, o bien para compartir música, videos, fotografías e información personal. Por ejemplo, Tuenti obtuvo 2.843.000 visitantes en el 2007, Hi5 registraba 2.279.000, en tanto, MySpace y Facebook registraban cerca de 1.200.000 visitantes únicos al mes (Watts, 2003). "A diferencia del modelo de LinkedIn y Xing, donde predomina la autenticidad de los perfiles publicados, las redes generalistas cuentan con infinidad de perfiles ficticios y con casi nulo control sobre los mismos", afirma Celaya (2008)

\section{El auge de las redes sociales especializadas}

Durante los últimos años, se ha registrado la aparición de redes sociales especializadas en una determinada actividad social o económica, un deporte o una materia. Esto permite satisfacer una necesidad inherente del ser humano de formar parte de grupos con características e intereses comunes, lo cual según la escala de necesidades de Maslow o pirámide de Maslow, obedecería a la necesidad de pertenencia o afiliación. Un ejemplo de este tipo de espacios es la red Flixster, que es una red social de amantes del cine, o bien SkiSpace, la cual está especializada en deportes de nieve, y las cuales han logrado atraer a grupos sociales interesados en estos temas, así como también, han captado la atención de anunciantes de acuerdo con cada segmento.

\section{Uso de la redes sociales en Educación}

Según (Borrás O. 2014., Fox B. 2017., Lucas E. 2015., Mccarroll N. 2015., Santiago R. 2013. \& Torres L. 2013) afirma que la uso de las redes sociales es como un resultado de las innovaciones tecnológicas se produce un desarrollo en cómo nos comunicamos de manera telemática y cómo evolucionan nuestras interacciones, apareciendo nuevos canales y formas de comunicación, además de una revolución en el acceso a la información.

Será necesario educar a estos estudiantes en competencias digitales para poder hacer frente a las tecnologías que hoy en día dominan el mercado y son un valor demandado en todo profesional. 
Gráfica 3: El uso del teléfono como herramienta de estudio.

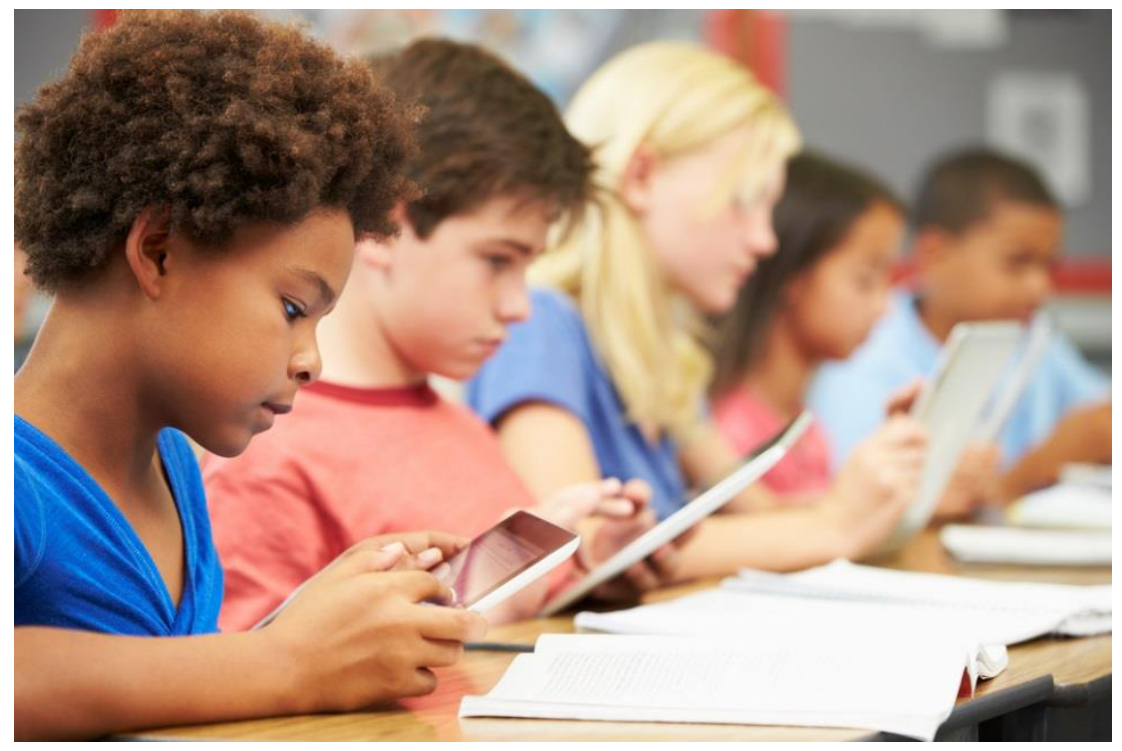

Autor: Lin McMak

Fuente:https://amp.review/wpcontent/uploads/2018/04/shutterstock_141206683.jpg

Aunque las generaciones más jóvenes integran estas tecnologías digitales dentro de su día a día de una manera natural, esto no implica que realmente sepan utilizarlas y las apliquen como una herramienta dentro de su formación. A estas generaciones se las denomina "Nativos digitales" y ha sido habitual, cada vez menos, asociarle de manera implícita un dominio de dichas tecnologías, hecho que se ha demostrado que no tiene relación alguna.

Las plataformas de redes sociales, hasta ahora vistas como algo asociado solo al ocio y algunas ocasiones a iniciativas personales o proyectos aislados de docentes o equipos docentes, van tomando peso en el campo educativo e incluso dentro del campo más puramente científico. Así lo revela la VIII Encuesta de Percepción social de la Ciencia del FECYT destacando Internet como segunda fuente de información científica para la población española tras la televisión, y dentro de Internet destacan las redes sociales. La prueba es que cada vez más organizaciones e instituciones se encuentran en estos medios, hecho que refuerza las redes sociales como elemento didáctico.

Es difícil no encontrar a día de hoy una Universidad que no cuente con al menos un perfil en alguna red social, y cada vez más servicios, departamentos o grupos de investigación se apoyen de dichas tecnologías, sin olvidar a los propios docentes que van usando estos espacios como fuente de información y como espacio de difusión. 
Ilustración 1: Los porcentajes del uso de las fuentes de información y comunicación donde se encuentran las redes sociales.

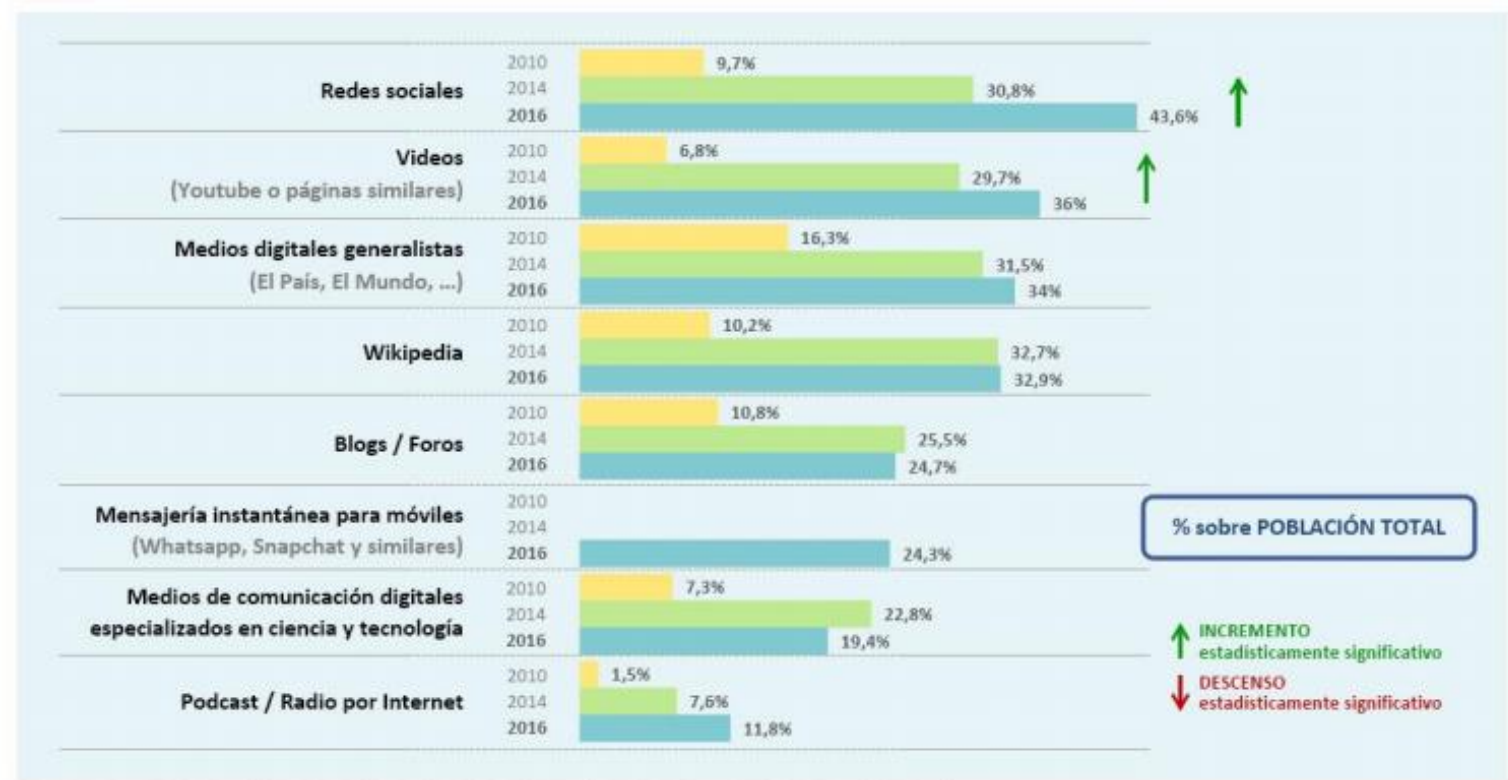

Recuperado de información científica en Internet.

Fuente: FECYT

Subido en: https://urjconline.atavist.com/uso-redes-sociales-educacion-1

Autor: Oril Borrás

Nosotros como docentes debemos conocer este tipo de tecnologías y aplicarlas en nuestras asignaturas, con una doble finalidad, desarrollar competencias digitales clave y dar una dimensión o valor superior a nuestras asignaturas, abriendo el aula y acercándonos a nuestros estudiantes. Sin olvidar el provecho personal como profesional y docente que supone un espacio como es Internet donde poder dar visibilidad a nuestro trabajo y favorecer las relaciones profesionales, además de aprender de otros.

\section{Definición}

El concepto de red social no es algo nuevo y representan las relaciones (trabajo, aficiones, parentesco, etc.) que se dan entre los seres humanos, surgiendo en diferentes contextos de la vida de un individuo. Se puede definir como una estructura social compuesta por un conjunto de personas que se relacionan entre sí, existiendo diferentes vínculos de relación y grados de estos. Evolucionado en el tiempo tanto las relaciones existentes como las personas que constituyen la red social.

Cuando el contexto se da en un espacio virtual, como puede ser Internet, y estas relaciones están soportadas por software instalado en un servido, se habla de plataformas, sitios o servicios de redes sociales, también se conocen como "redes sociales virtuales". Estos servicios están construidos aprovechando las ventajas que ofrece la Web 2.0, entendiéndola como una evolución de Internet como espacio en el que los individuos no solo consumen información, sino que también la generan y son activos en esta. 
Todo individuo que participa en Internet automáticamente pasa a tener una identidad digital, una representación del individuo como resultado de todo lo que hace en la red, lo que se conoce como huella digital, creada tras su paso por Internet. Toda acción asociada a su persona construirá esta identidad, desde un inocente comentario en un blog hasta fotografías subidas o cualquier tipo de publicación, incluso la actividad de terceros si mencionan a dicha persona. Asociada a la identidad digital encontramos la reputación online que hace referencia a cómo nos ven terceros y es un aspecto totalmente subjetivo de nuestra identidad, y por otro lado la visibilidad o lo conocido que somos, aquella suma de acciones asociadas a nuestra identidad digital que indican el grado de una manera objetiva de presencia que tenemos en la red.

Esta idea está en conjunción con la cultura promovida de la web 2.0 de colaboración y participación, fomentando el trabajo grupal.

Las plataformas de redes sociales permiten a los individuos construir perfiles públicos o privados que representen su identidad digital y conectar con otros usuarios, sean conocidos o no, para interactuar a través de publicaciones de cualquier tipo, habitualmente texto, pero también imagen, vídeo, audio, recopilaciones de enlaces a páginas web, etc.

Se empieza a hablar de términos como "universidades 2.0" con una finalidad social en la que colaborar a través de las herramientas que ofrece la Web, creando espacios comunes en los que interactuar y generar sinergias entre docentes, investigadores, alumnos, profesionales y en general todo el conjunto de la sociedad.

Redes sociales y cómo nos pueden ayudar en el aula, en este tema se analizará cómo aplicar en nuestra asignatura redes sociales, para crear una comunidad virtual de aprendizaje que complemente y ayude a abrir el aula.

Aplicar nuevas metodologías en la educación debe buscar un fin y estar incluido dentro de un diseño instruccional, no por el hecho de apostar por nuevas tecnologías.

El primero de los pasos será siempre, desde un punto de vista didáctico, analizar en qué podrá beneficiar la creación de una comunidad virtual de aprendizaje tanto a nuestro alumno como al funcionamiento de la asignatura. Por lo tanto ver nuevas posibilidades que puedan ofrecer, por ejemplo acercamiento a profesionales, desarrollo de competencias digitales en el estudiante o detectar aquellas carencias, como por ejemplo falta de motivación, comunicación o de interacción en el aula redes.

\section{Ministerio de Educación recomienda evitar uso de redes sociales para comunicación entre profesores y estudiantes}

El uso de la tecnología en las aulas es común en nuestros días, ya que sirve para complementar los conocimientos de los estudiantes. En las clases, lo digital tiene un peso. Pero la utilización de las redes sociales como medio de comunicación entre alumnos y docentes no está permitida. Así detalló el Ministerio de Educación en un correo 
electrónico, a través del que respondió a este Diario. A través del mismo se explicó que no aconseja el uso de redes sociales como Facebook, Twitter, Instagram, WhatsApp y otras, para que profesores y estudiantes se comuniquen. "Al ser de libre uso pueden traer consecuencias negativas para los estudiantes. Se recomienda que las tareas escolares sean planificadas y socializadas en clase, de tal manera que se evite utilizar este tipo de redes sociales para este propósito". Sin embargo, el empleo de otras herramientas y plataformas pueden enriquecer las clases. Por ejemplo, en el área de Lengua y Literatura se fomenta el uso de soportes digitales para fortalecer la comunicación oral y escrita, la consulta de bases de datos, entre otros recursos. Aquí el papel del docente es fundamental, ya que ayuda a discernir el tipo de información. Además se enseña a “...valorar la confiabilidad de las fuentes escogidas y la cita respectiva para fomentar en el estudiante la honestidad académica, entre otros conocimientos". Hasta agosto del 2018 se han registrado 27 denuncias de contacto físico con finalidad sexual contra menores de 18 años vía medios electrónicos. Los datos nacionales fueron proporcionados por la Unidad de Violencia de Género de la Fiscalía del Guayas. Adicionalmente, el Consejo de la Judicatura indicó que se dictaron 63 sentencias por esta causa en los últimos tres años. Este delito conocido como 'grooming' o acoso sexual vía Internet sí está tipificado en el Código Orgánico Integral Penal (COIP). La sanción es de uno a cinco años de prisión. (Heredia, 2018, pág. $1)$.

Gráfica 4: La utilización de las redes sociales como medio de comunicación entre alumnos y docentes no está permitida.

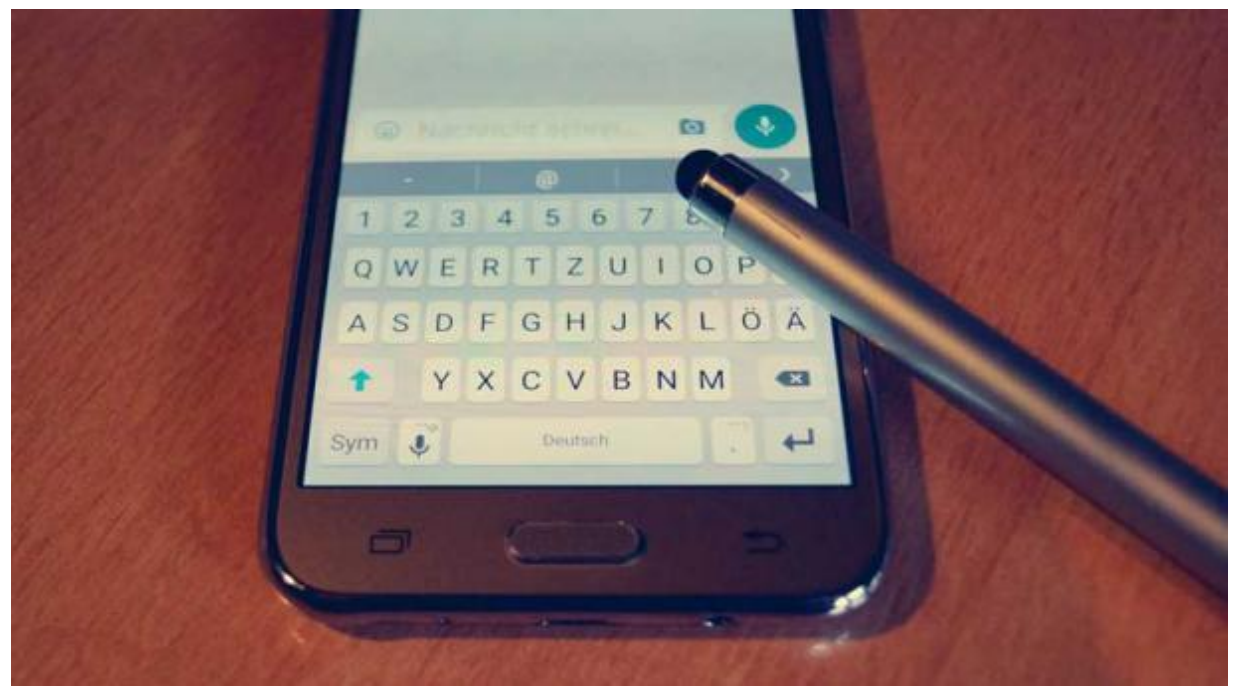

Autor: Daniela Cevallos.

Fuente:https://www.elcomercio.com/files/article_main/uploads/2018/10/15/5bc 509e87fd4f.jpeg

En la actualidad podemos contar con muchas redes sociales que contribuyen al intercambio fácil de cualquier tipo de información en diversos formatos, como lo son Facebook, Twitter, Google +, likedIn, Instagram, por no mencionarlos todos. 
En este caso hablaremos de Facebook, la red social por excelencia, ya que ésta es una herramienta muy popular y de fácil manejo; esta red no esta diseñada para educar como tal, pero sí es un medio que genera mucho más aprendizaje, debido a que es una herramienta online que esta al alcance de cualquier persona sea por medio de una Laptop, un Smartphone o Tablet.

Con la ayuda de las redes sociales, principalmente de Facebook, los docentes podrán generar foros donde ellos mismos pongan las reglas de participación, así los alumnos podrán resolver las dudas que tengan sobre cierto tema en cualquier momento. También pueden crear un grupo privado donde se incluyan a todos los alumnos a los que el docente imparte clase y así poder administrar un solo espacio educativo donde se fomente el aprendizaje colectivo. Lo mejor de Facebook es que puedes seguir en tiempo real la participación de los demás, ya que te llegan las notificaciones directamente a tu correo electrónico, o a tu dispositivo móvil.

Los sistemas educativos de todo el mundo están experimentando una revolución en la enseñanza y el aprendizaje. Con la llegada y el establecimiento de las nuevas tecnologías, se han creado nuevas vías de comunicación e interacción entre alumnos, profesores y el resto del mundo impulsado por la web. El aprendizaje digital y social a menudo se basa en el hecho de que los jóvenes tienen acceso a los conocimientos y al aprendizaje por ellos mismos. Ese aprendizaje es típicamente interactivo, centrado en el estudiante, colaborativo e instantáneo. Se realiza fuera del horario escolar, en contextos no formales y cada vez más entre amigos y a través de redes sociales. La enseñanza y el aprendizaje son y serán mucho más sociales.

Los jóvenes de hoy también tienen la habilidad de comunicarse con cualquier persona alrededor del mundo utilizando una gran variedad de plataformas digitales, principalmente con teléfonos móviles y tabletas. Estas herramientas permiten a millones de personas conectarse y compartir información de una manera sin precedentes. Se han planteado interrogantes acerca de cómo preparar mejor a los jóvenes para un mundo digital y la necesidad de una alfabetización digital y las habilidades necesarias para el mundo laboral. Lo que creemos es que hay que dar a los jóvenes acceso a las plataformas digitales en escuelas, institutos y otros entornos de aprendizaje para que les proporcione habilidades básicas y esenciales que les permitan navegar dentro de su mundo digital y que les permitan ser ciudadanos digitales preparados. (Basterrechea, 2015, pág. 5) 
Gráfica 5: El modo de implementación de la red social en la educación.

\begin{tabular}{|c|c|c|}
\hline Aprendizaje formal & $\begin{array}{l}\text { Aprendizaje informal fuera del } \\
\text { horario escolar }\end{array}$ & Aplicaciones más amplias \\
\hline $\begin{array}{l}\text { La creación de una Biografía o } \\
\text { Grupo de Facebook para apoyar } \\
\text { la enseñanza de cualquiera } \\
\text { material curricular } \\
\text {. La creación de un espacio y una } \\
\text { plataforma para publicar los } \\
\text { deberes o recursos recursos } \\
\text { para repasar } \\
\text { Organizar debates sobre } \\
\text { temas de actualidad en los } \\
\text { medios de comunicación } \\
\text { Tutoría entre compañeros } \\
\text { y asistencia } \\
\text { Una herramienta de } \\
\text { investigación para publicar, } \\
\text { compartir ideas, videos y } \\
\text { recursos } \\
\text { La creación de grupos en las } \\
\text { escuelas para facilitar el trabajo } \\
\text { de los profesores y personal } \\
\text { docente }\end{array}$ & $\begin{array}{l}\text { La organización de un equipo } \\
\text { deportivo o una actividad } \\
\text { extra-escolar } \\
\text { - Facilitar la adaptación de los } \\
\text { alumnos nuevos a las escuelas } \\
\text { y los colegios } \\
\text { - Lacreación y el diseño de } \\
\text { actividades digitales como la } \\
\text { creación de aplicaciones } \\
\text { Organización de reuniones de } \\
\text { profesores y otras actividades } \\
\text { del claustro } \\
\text { El apoyo informal de amigos } \\
\text { (dar me gusta) para proyectos } \\
\text { y otras actividades } \\
\text { Publicación de podcasts } \\
\text { sociales y de vídeos para los } \\
\text { estudiantes y compañeros } \\
\text { Creación de grupos para los } \\
\text { profesores a través de una } \\
\text { facultad o federación de } \\
\text { escuelas, colegios, } \\
\text { universidades }\end{array}$ & $\begin{array}{l}\text { Una herramienta de } \\
\text { comunicación con los padres, } \\
\text { los cuidadores y la comunidad } \\
\text { - Facilitar a los estudiantes de } \\
\text { idiomas la conversación con } \\
\text { compañeros de intercambio } \\
\text { en el extranjero } \\
\text { - Participar y llegar a los } \\
\text { estudiantes en las escuelas, } \\
\text { las universidades y a través del } \\
\text { aprendizaje en línea } \\
\text { - Proporcionar inspiración y } \\
\text { formación de habilidades para } \\
\text { la vida y otras materias } \\
\text { - La enseñanza de habilidades } \\
\text { digitales para jóvenes y adultos } \\
\text { - Involucrar a los jóvenes en la } \\
\text { juventud y en la comunidad } \\
\text { Capacitar a los estudiantes para } \\
\text { socializar y hacer amigos }\end{array}$ \\
\hline
\end{tabular}

Autor: Natalia Basterrechea.

Fuente:http://www.ednfoundation.org/wpcontent/uploads/Facebookguidespanish.pdf

\section{Estadísticas de redes sociales en Ecuador 2017}

Las agencias Misiva, Laika, Ximah, Agencia Paradais, Kick Ads, y Geeks, son las fundadoras de ésta nueva era para IAB Ecuador.

Se han unido principalmente para mejorar y estructurar lo que debería ser la industria digital a futuro.

Por eso motivo fue presentado el estudio de Estadisticas de redes sociales en Ecuador 2017 y consumo de medios digitales. Liquid LAB por parte de Misiva junto con Netquest realizaron el estudio a principios de este año. Podemos encontrar que Facebook sigue manteniendo el liderazgo en cuanto a consumo del mismo y Whatsapp está muy de cerca. 
Ilustración 2: EL uso de las redes sociales según su aplicación.

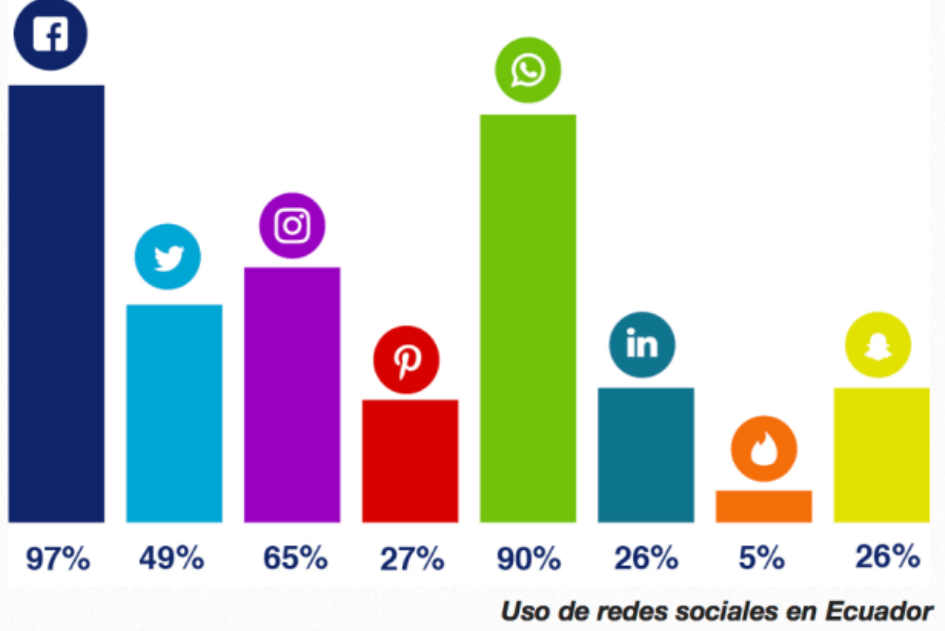

Autor: Mauricio Arboleda.

Fuente: https://i2.wp.com/www.hablemosdemarcas.com/wpcontent/uploads/2017/12/redes-sociales-en-Ecuador2017.png?resize $=768 \% 2 \mathrm{C} 504$

Ilustración 3: El uso de Instagram entre 14-23 años es bastante grande con respecto a la 24-59 años de edad..

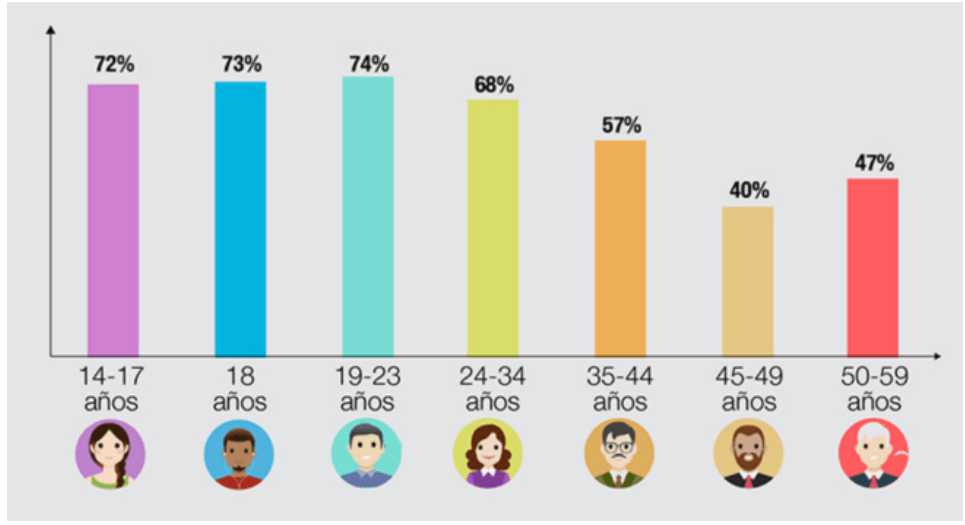

Autor: Mauricio Arboleda.

Fuente: https://i2.wp.com/www.hablemosdemarcas.com/wpcontent/uploads/2017/12/instagram-redes-sociales-en-Ecuador2017.png?resize $=768 \% 2 \mathrm{C} 417$ 
Ilustración 4: El móvil sigue siendo el dispositivo más usado durante el día, aunque tiene caídas significativas en horarios laborales.

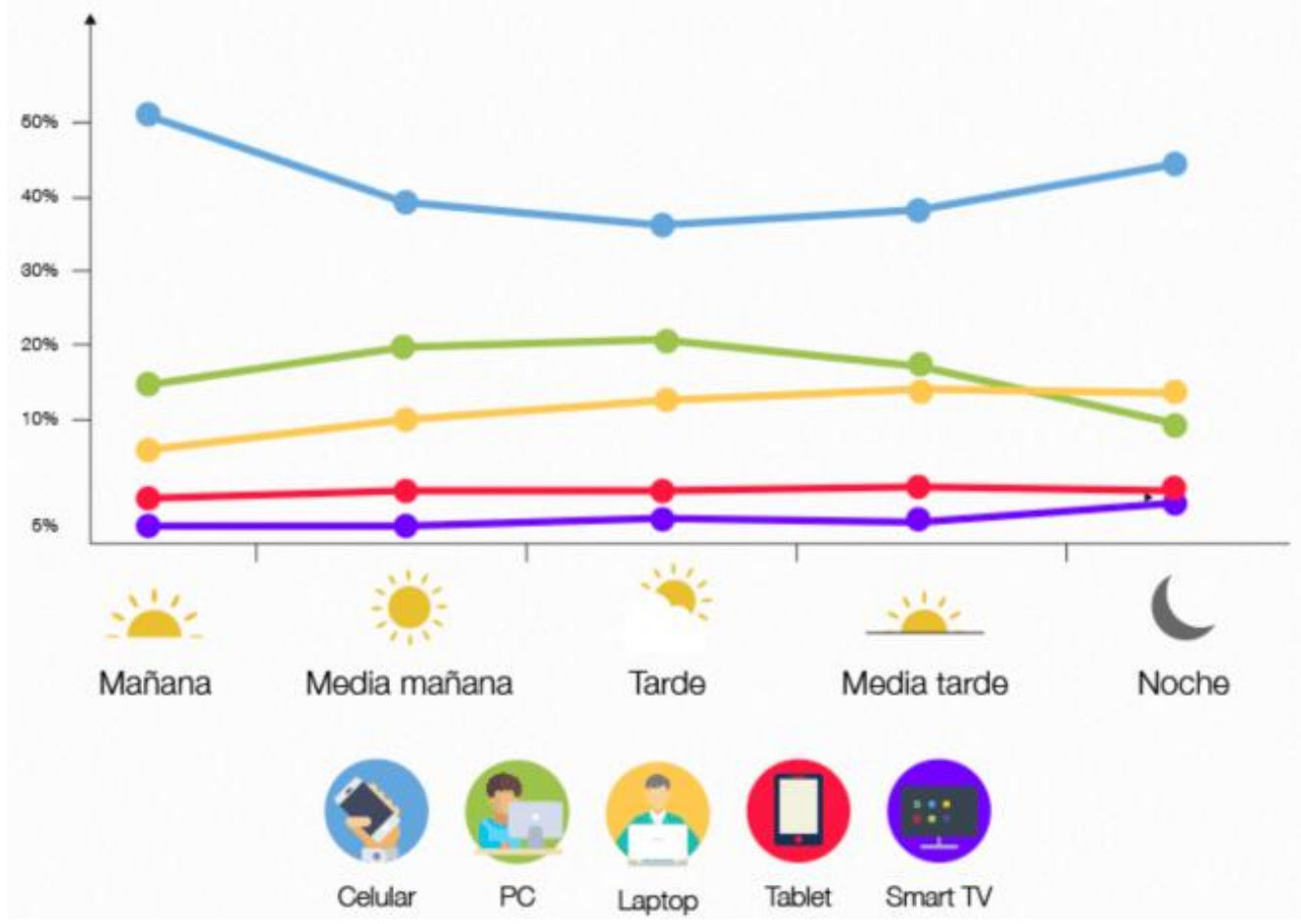

Autor: Mauricio Arboleda.

Fuente:https://i0.wp.com/www.hablemosdemarcas.com/wpcontent/uploads/2017/12/dispositivos-estadisticas-de-redes-sociales-en-ecuador2017.png? resize $=768 \% 2 \mathrm{C} 545$

También se menciona que Facebook y Youtube realizan una batalla campal sobre quien tiene mayor cantidad de reproducciones. Recordemos que la red social viene trabajando mucho en su algoritmo que le ha perjudicado el tráfico web haya disminuido para las marcas. (Arboleda, 2017).

En el sistema que da el mundo a cada uno de los países es beneficioso para algunos y perjudicial para otros. En este caso podemos ver el porcentaje de las utilizaciones de las redes sociales a nivel mundial y en nuestro país Ecuador. Donde el consumo del internes es esencial para la comunicación investigación ya que todo está en la red.

\section{Conclusiones}

Las redes sociales es una de las fuentes de comunicación más usada a nivel mundial donde los niños, adolescentes, jóvenes y adultos las utilizan con mayor frecuencia cada día. 
$>$ El modo de utilizar una red social puede ser de un sin número de variables como el ámbito educativo, comercial, comunicación, publicidad donde las empresas pequeñas, medias y grandes las utilizan para promocionar su mercado.

$>$ En la educación los docentes tienen la facilidad de comunicarse con sus alumnos para adquirir una información, él envió o entrega de tareas o como también clases online para personas que no dispongan de tiempo completo.

$>$ Es bien saber que la red social es la moda actual, donde también se ha visto caso de acoso sexual entre docentes a estudiantes que a nivel mundial se a logrado captar los hechos de tales abusos.

\section{Referencias Bibliográficas}

Alejandra, S. (26 de 06 de 2015). ¿Quién invento las redes sociales? Obtenido de prezi: https://prezi.com/settjf2cdiqx/quien-invento-las-redes-sociales/

Arboleda, M. (18 de 12 de 2017). Estadísticas de redes sociales en Ecuador 2017. Obtenido de http://www.hablemosdemarcas.com/estadisticas-de-redes-socialesen-ecuador-2017/

Basterrechea, N. (12 de 03 de 2015). Guía de Facebook para Educadores. Obtenido de Una heramienta para enseñar y aprender: http://www.ednfoundation.org/wpcontent/uploads/Facebookguidespanish.pdf

Borrás, O. (07 de 08 de 2014). Uso de las Redes sociales en Educación. Obtenido de https://urjconline.atavist.com/uso-redes-sociales-educacion-1

Fernández, R. (2013). Redes sociales, apoyo social y salud . BARCELONA: PERÍFERIA. Obtenido de http://revistaredes.rediris.es/Periferia/english/number3/periferia_3_3.pdf

Flores, J. (01 de 10 de 2014). Las redes sociales. Universidad de San Martín de Porres . PERU: UVA . Obtenido de http://mc142.uib.es:8080/rid\%3D1HY8TVCBB15599LW-1S6Z/redes_sociales.pdf

Fox, B. (22 de 08 de 2017). Social Media and Education: Using Social Media For Better Academic Results. Obtenido de https://medium.com/@braydenfox/social-mediaand-education-using-social-media-for-better-academic-results-804b7a41a825

Harold, H. (2014). LAS REDES SOCIALES: UNA NUEVA HERRAMIENTA DE DIFUSIÓN. 4-5. doi:http://www.redalyc.org/pdf/729/72923962008.pdf

Heredia, V. (15 de 10 de 2018). Ministerio de Educación recomienda evitar uso de redes sociales para comunicación entre profesores y estudiantes. EL COMERCIO, pág. 1. Obtenido de https://www.elcomercio.com/actualidad/educacion-redessociales-profesores-alumnos.html 
Lucas, E. (08 de 09 de 2015). Redes sociales en el aprendizaje moderno. Obtenido de http://www.finanzaspersonales.co/columnistas/articulo/educacion-cual-es-eluso-de-las-redes-sociales-en-la-educacion/76065

Mccarroll, N. (07 de 2015). Social Networking in Education. Obtenido de https://www.researchgate.net/publication/269558511_Social_Networking_in_Ed ucation

Requena, F. (2013). EL CONCEPTO DE RED SOCIAL. MÁLAGA: UNIVERSIDAD DE MÁLAGA. Obtenido de file://C:/Users/Valencia/Downloads/DialnetElConceptoDeRedSocial-249260.pdf

Rouse, M. (30 de 10 de 2017). Social network. Obtenido de https://www.computerhope.com/jargon/s/socinetw.htm

Santiago, R. (5 de 11 de 2013). Las razones para usar las redes Sociales. Obtenido de https://www.theflippedclassroom.es/7-razones-para-hacer-uso-de-la-redessociales-en-educacion/

Torre, L. d. (2012). Las redes sociales: conceptos y teorías. Argentina: Consonancias. Obtenido de http://bibliotecadigital.uca.edu.ar/repositorio/revistas/redes-socialesconceptos-teorias.pdf

Torres, I. (2013). Uso de las redes sociales como estrategias de aprendizaje. ¿Transformación Obtenido de http://www.udgvirtual.udg.mx/apertura/index.php/apertura/article/view/198/213

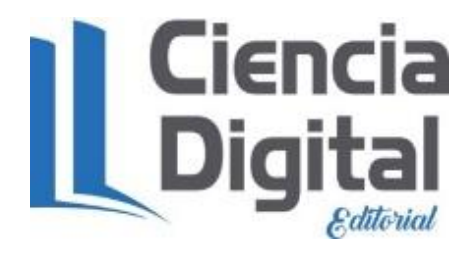




\section{Para citar el artículo indexado.}

Velasteguí E. (2018). El uso de las redes sociales como estrategia educativa. Revista electrónica Explorador Digital 2(4), 64-79. Recuperado desde:

http://cienciadigital.org/revistacienciadigital2/index.php/exploradordigital/article/view/341/7 53

\section{Ciencia \\ Digital \\ Editorial}

El artículo que se publica es de exclusiva responsabilidad de los autores y no necesariamente reflejan el pensamiento de la Revista Explorador Digital.

El articulo queda en propiedad de la revista y, por tanto, su publicación parcial y/o total en otro medio tiene que ser autorizado por el director o editor de la Revista Explorador Digital.
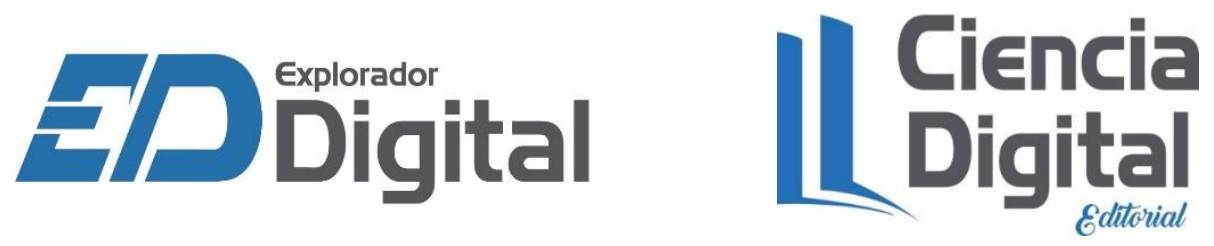